\title{
Nanocoated implant surfaces; a short overview
}

\begin{abstract}
Surgeons are continuously in search of slightly invasive methods to treat their patients. One of the chief goals of nanotechnology in surgery is designing of such invasive procedures. Nano-based coatings have been developed that can significantly improve the properties of surgical implants like wear features, fixation and biocompatibility. A short review process was performed using internet database by means of Mesh keywords such as implant, coatings, nano particles and nanotechnology. The searches included full-text publications written in English without time limitation. The reviewed literature revealed that nano-based implant surfaces can significantly impact on the growth and proliferation of cells in helpful ways.
\end{abstract}

Keywords: implant coatings, nanoparticles, nanotechnology, nano-hydroxyapatite, hydroxyapatite coatings
Volume 6 Issue I - 2018

\author{
Ali Reza Karimpour,, ${ }^{1,2}$ Solmaz Maleki Dizaj,3 \\ 'ISACOTechnical Training Center, Iran \\ 2Matin-Plast Company, Iran \\ ${ }^{3}$ Dental and Periodontal Research Center, Tabriz University of \\ Medical Sciences, Iran \\ ${ }^{4}$ Student Research Committee, Tabriz University of Medical \\ Sciences, Iran
}

Correspondence: Solmaz Maleki Dizaj, Tabriz University of Medical Sciences, Golgasht Street, Daneshgah Ave., Tabriz, Iran, Tel +98(4I) 3337 2250, Email maleki.s.89@gmail.com

Received: February 18, 2018 | Published: March 05, 2018

\section{Introduction}

An implant is an artificial object with ability to replace or stabilize injured body functions and it can be in various kinds. Titanium, cement and polymers are used to for producing of implants in biocompatibility enhancing way. ${ }^{1,2}$ Implants have been effectively used for the replacement of orthopedic/dental components in the treatment of total or partial bone defects with the acceptable success rates. However, current orthopedic/dental implants sometimes suffer from a limited lifetime due to implant failure. According to reports, there are various reasons for implant failures such as stress and strain imbalance between implant and its surround, poor primary bone growth on implant surface as well as generation of wear debris in articulating components of implant. The investigator's studies show that failure rate can be reduced if implant material stimulates fast creation of new bone. Enhancement of implant's fixation is also can be decreased the failure rate. ${ }^{1,3}$

Nanoparticles are defined as ultrafine particles in nanometer ranges, and are prepared from mostly any type of biocompatible material. ${ }^{4,5}$ Recently, they have been used to improve bone incorporation of bone related implants. ${ }^{1,6-10}$ Nanoparticles have also been applied as coatings materials to implant surfaces to progress soft tissue integration and increase implants success rate. Based on recent studies, new coatings at a nano range can improve the wear characteristics, fixation and biocompatibility of surgical implants. Similarly, nano-coated implants can importantly influence the growth and proliferation of cells. Numerous recent studies have shown the outstanding properties of different implant coating materials such as nano structured diamond, nano-hydroxyapatite and metallic nanoparticles. ${ }^{1,3}$

It has been reported that nano structured diamond coatings show good adhesion to titanium based implants. According to some studies, life time of orthopedic implants could increase to upwards of 40 years. ${ }^{1,3}$

Osteoconductive nano materials as coatings of implants can induce a chemical bond with bone and achieve proper biological fixation. It is discovered that coating of bioinert nano particles (e.g., zirconium and aluminum) on Ti implant surfaces may alter the physical possessions and also, improve the osteogenic potential of implants. It has been also informed that materials like titanium nano tubes are able to enhance the density of osteoblast cells on the implant that can present better implant stability. $3,11,12$

The investigations shows that hydroxyapatite coatings are more believed for dentistry than in orthopedics, but its potential is high in both areas. Hydroxyapatite indorses bone formation around the implant, enhance osteoblast (bone-forming cell) functions such as adhesion, proliferation and mineralization. ${ }^{1,3}$

\section{Conclusion}

Recently, nano-coatings have been described to have helpful possessions to improve implant fixation and increase the success rate. Nanoparticles are applied as particle coatings on the implant surface with ability to increase the functionality and the stability and fixation of the implant.

\section{Acknowledgments}

None.

\section{Conflict of interest}

Authors declare there is no Conflict of interest towards this manuscript.

\section{References}

1. Parnia, F, Yazdani J, Javaherzadeh V, et al. Overview of nanoparticle coating of dental implants for enhanced osseointegration and antimicrobial purposes. Journal of Pharmacy \& Pharmaceutical Sciences. 2017;20:148-160.

2. h t t p s : / / p d f s. s e ma n t i c s c ho 1 a r.org aefe/3decdeb5af2686b4a89fb288d9a3e79ab7a8.pdf

3. Weldon C, Tian B, Kohane DS, et al. Nanotechnology for surgeons. Wiley Interdisciplinary Reviews:Nanomedicine and Nanobiotechnology. 2011;3:223-228.

4. Salatin S, Jelvehgari M, Maleki Dizaj, et al. A sight on protein-based nanoparticles as drug/gene delivery systems. Therapeutic delivery. 2015;6:1017-1029. 
5. Dizaj SM, Barzegar Jalali M, Maleki Dizaj S, et al. Calcium carbonate nanoparticles; potential in bone and tooth disorders. Pharmaceutical Sciences. 2015;20:175-182.

6. Dizaj S.M, Lotfipour F, Zarrintan, M.H, et al. Physicochemical characterization and antimicrobial evaluation of gentamicin-loaded $\mathrm{CaCO} 3$ nanoparticles prepared via micro emulsion method. Journal of Drug Delivery Science and Technology. 2016;35:16-23.

7. Maleki Dizaj S, Lotfipour F, Barzegar-Jalali, et al. Application of Box-Behnken design to prepare gentamicin-loaded calcium carbonate nanoparticles. Artificial cells, nanomedicine, and biotechnology. 2016;44:1475-1481.

8. Maleki Dizaj S, Lotfipour F, Barzegar-Jalali, et al. Ciprofloxacin $\mathrm{HCl}$-loaded calcium carbonate nanoparticles: preparation, solid state characterization, and evaluation of antimicrobial effect against Staphylococcus aureus. Artificial cells, nanomedicine, and biotechnology. 2017;45(3):535-543.
9. Maleki Dizaj S, Lotfipour F, Barzegar-Jalali, et al. Ciprofloxacin $\mathrm{HCl}$-loaded calcium carbonate nanoparticles: preparation, solid state characterization, and evaluation of antimicrobial effect against Staphylococcus aureus. Artificial cells, nanomedicine, and biotechnology. 2017;45(3):535-543.

10. Sharma S, Ruffenach G, Umar S, et al. Role of oxidized lipids in pulmonary arterial hypertension. Pulmonary circulation. 2016;6(3):261273.

11. Barboni B, Mangano C, Valbonetti, L, et al. Synthetic Bone Substitute Engineered with Amniotic Epithelial Cells Enhances Bone Regeneration after Maxillary Sinus Augmentation. Plos One. 2013;8(5).

12. Chien CY, Liu T.Y, Kuo, W.H, et al. Dopamine-assisted immobilization of hydroxyapatite nanoparticles and RGD peptides to improve the osteoconductivity of titanium. Journal of Biomedical Materials Research. 2013;101:740-747. 